\title{
Active decoupling of RF coils using a transmit array system
}

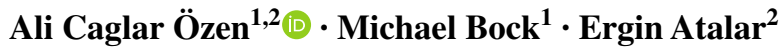

Received: 14 April 2015 / Revised: 16 July 2015 / Accepted: 17 July 2015 / Published online: 5 August 2015

(C) ESMRMB 2015

\begin{abstract}
Objective Implementation of a decoupling method for isolation of transmit and receive radio frequency (RF) coils for concurrent excitation and acquisition (CEA) MRI in samples with ultra-short $\mathrm{T}_{2} *$.

Materials and methods The new phase and amplitude (PA) decoupling method is implemented in a clinical 3T-MRI system equipped with a parallel transmit array system. For RF excitation, two transmit coils are used in combination with a single receive coil. The transmit coil is geometrically decoupled from the receive coil, and the remaining $B_{1}$-induced voltages in the receive coil during CEA are minimized by the second transmit coil using a careful adjustment of the phase and amplitude settings in this coil. Isolation of the decoupling scheme and transmit noise behavior are analyzed for different loading conditions, and a CEA MRI experiment is performed in a rubber phantom with sub-millisecond $\mathrm{T}_{2}{ }^{*}$ and in an ex vivo animal.

Results Geometrical $(20 \mathrm{~dB})$ and PA decoupling $(50 \mathrm{~dB})$ provided a total isolation of $70 \mathrm{~dB}$ between the transmit and receive coils. Integration of a low-noise RF amplifier was necessary to minimize RF transmit noise. CEA MR
\end{abstract}

Part of this document was presented and defended as a Master of Science Thesis at Bilkent University, Department of Electrical and Electronics Engineering, in May 2013 by A.C. Ozen under the supervision of Prof. E. Atalar.

Ali Caglar Özen

ali.oezen@uniklinik-freiburg.de

1 Department of Radiology, Medical Physics, University Medical Center Freiburg, 79106 Freiburg, Germany

2 Department of Electrical and Electronics Engineering, Bilkent University, 06800 Ankara, Turkey images could be reconstructed from a rubber phantom and an ex vivo animal.

Conclusion CEA MRI can be implemented in clinical MRI systems using active decoupling with parallel transmit array capabilities with minor hardware modifications.

Keywords Geometrical decoupling $\cdot$ Phase amplitude decoupling · Transmit array · Continuous-wave NMR . Concurrent excitation and acquisition

\section{Introduction}

In this work, a novel method for decoupling of transmit and receive coils is proposed that can be used to implement magnetic resonance imaging (MRI) with concurrent excitation and acquisition (CEA). In conventional MRI systems, time interleaved excitation and acquisition is used, and transmit and receive circuitry are separated electronically by transmit-receive (T/R) switches and detuning circuits. With the growing interest in MRI of tissues with short $\mathrm{T}_{2}$ * components such as bones, cartilage, tendons, and lungs, currently used interleaved MRI techniques and hardware introduce unwanted dead times between RF excitation and data acquisition [1].

Ultimately, the dead time (or acquisition delay) between the end of RF excitation and the start of data acquisition sets a limit to the lowest achievable echo time (TE) or results in missing data points at the center of $\mathrm{k}$-space. Major sources of dead time are T/R switching, analog-to-digital converter (ADC) filtering, and the coil ring down time [2], which can amount to $40 \mu \mathrm{s}$ or more in clinical MRI systems. Dead times can be reduced significantly by special hardware [3], and there are time-interleaved acquisition techniques that use strong gradient fields to resolve signals from short $\mathrm{T}_{2}$ * 
materials [4], and pure phase encoding techniques which utilize rather moderate gradient strengths $[5,6]$. Although line broadening effects of short $\mathrm{T}_{2}{ }^{*}$ samples reduced with increasing maximum gradient strength, hardware limitations such as the acquisition delay still impose a lower limit on the shortest detectable $\mathrm{T}_{2}{ }^{*}$ value. However, CEA is an alternative approach where radio frequency (RF) excitation and data acquisition are performed concurrently.

CEA was historically the first MR technique, which at that time was called continuous-wave $(\mathrm{CW})$ nuclear magnetic resonance (NMR). It has been extensively used to spectroscopically investigate solid materials with ultrashort $\mathrm{T}_{2}{ }^{*}[7,8]$, and has been adapted to ${ }^{1} \mathrm{H}$ and multinuclear imaging to utilize the fact that CW NMR mitigates the dead time between RF excitation and signal acquisition [9-12]. Although outdated, CEA remains an active area of research, because it has advantages over interleaved NMR methods: CEA allows for the detection of structures with ultrashort relaxation times, and the peak RF power can be reduced to the order of one hundred milliwatts. Although CEA has been applied to study industrial products and solid state materials with very short $\mathrm{T}_{2}{ }^{*}$, it can potentially also be used in in vivo studies, in which interleaved techniques such as ultra-short echo time (UTE) or sweep imaging with Fourier transform (SWIFT) techniques are limited. In interleaved sequences, signals decaying more rapidly than the system dead times cannot be detected, whereas CEA continuously monitors the MR signal even during RF excitation. Thus, tightly bounded water in bone, for example, is not visible with interleaved NMR approaches [13-17], and new contrast mechanisms and novel sequences may be developed with further research on CEA.

Methods proposed for the implementation of true CEA include geometric decoupling [18], sideband excitation [19], and hybrid coupler isolation [20, 21]. In geometric decoupling, separate transmit and receive coils are placed orthogonally to each other around the imaging volume. With this orthogonal setup, the cross-talk between transmit and receive coil is minimized so that the unwanted RF excitation signal is highly suppressed during data acquisition. Sideband excitation uses off-resonant excitation on the order of a few megahertz, and filtering in the time domain. However, off-resonant excitation increases the RF power that is required to achieve flip angles comparable with onresonant excitation. In addition, the sample will experience an unwanted Bloch-Siegert shift during off-resonant excitation, and the hardware required can be complex in clinical applications. Alternatively, a hybrid coupler system can be connected to the RF coils to suppress the excitation field below the dynamic range of the receiver. The hybrid coupler has a phase difference of $180^{\circ}$ between the RF input port and the output port to the receiver circuitry. The hybrid coupler subtracts the acquired signal, which is the additive combination of the MR signal and the RF excitation from the input RF excitation signal. Due to non-idealities in the circuit and the frequency dependency of the hybrid coupler, the unwanted RF excitation signal often leaks through to the receiver due to the limited isolation (maximum of $30-40 \mathrm{~dB}$ isolation is reported in Idiyatullin et al.). Thus, the MR signal needs to be extracted from the acquired data by a cross-correlation technique. Hybrid coupling has additional disadvantages: (1) the need for high dynamic range receiver electronics, (2) extremely accurate tuning of the isolator, which is sensitive even to small changes in coil impedance, and (3) contaminations of the acquired signal by imperfections of the RF excitation field.

In this study, CEA is implemented by combining geometric decoupling with a novel method that adjusts the relative phase between two separate transmit coils. This method attempts to cancel the $B_{1}$-induced currents on the receive coil by an appropriate adjustment of the amplitudes and phases of the transmit coil array input currents [22, 23]. The method was tested for CEA imaging of a short- $\mathrm{T}_{2} *$ phantom and an ex vivo mouse in a 3T clinical MRI system.

\section{Theory}

\section{Decoupling of RF coils with additional decoupling coils}

It was recently shown that a parallel transmit array system can be used to cancel RF currents that are induced on a bare wire [24]. In this work, this concept is adapted to cancel the $\mathrm{RF}$ currents that are induced on a loop receive coil. In general, the combined RF field generated by a set of transmit RF coils induces a current in a resonant receive coil, which is observed at the output of the receive coil as a $B_{1}$-induced voltage. Using an additional set of transmit coils, which are called decoupling coils, it is possible to cancel the $B_{1}$ induced currents in the receive coil by adjusting their RF phases and amplitudes. For a transmit array system with $N$ coils, some of which are used as transmit coils and some as decoupling coils, the total current $I$ induced in a receiver coil can be expressed as:

$I=\sum_{n=1}^{N} a_{n} I_{n}=0$

Here, $I_{n}$ is the $B_{1}$-induced current, and $a_{n}$ is an arbitrary complex coupling coefficient for the $n$th transmitting coil, which represents the geometric and electromagnetic factors that affect the amount of coupling between the receive coil and the $n$th transmit coil.

In its simplest form, the phase and amplitude (PA) decoupling system can be implemented with two transmit coils—one to apply the RF excitation, the other for 
decoupling. By adjusting the amplitude and phase $a_{2}$ of the decoupling coil, one can theoretically achieve a cancellation of the total current in the receive coil: $a_{1} I_{1}+a_{2} I_{2}=0$. In practice, it is sufficient to measure the voltages that are induced by each of the transmit coils individually, and to solve

$V_{1}+c V_{2} e^{j \theta}=0$

for the two parameters $c$ and $\theta$.

\section{Application to MRI with CEA}

A CW-NMR experiment can be modeled as a linear system in which the input is the $B_{1}$ excitation signal and the output is the convolution of the system response (FID) and the input $B_{1}$, which is a chirp signal that covers a certain frequency range [4]. For short $\mathrm{T}_{2} *$ values and small tip angles, approximations can be used to account for the linearity condition during CW excitation [5]. In a CEA experiment, the acquired MR signal, $s(t)$, can be written as an additive combination of the convolution signal and a frequencydependent leakage component $A(t)$, which is the remaining $B_{1}$-induced voltage due to imperfect decoupling:

$$
\begin{aligned}
& s(t)=F I D \otimes B_{1}(t)+A(t) \\
& s(t)=F I D \otimes B_{1}(t)+h(t) \otimes B_{1}(t) \\
& B_{1}(t)=e^{-j \pi f_{\mathrm{s}} / t_{\mathrm{acq}} t^{2}}
\end{aligned}
$$

The symbol $\otimes$ stands for the convolution operator. The leakage can be determined experimentally by a system response $h(t)$, which is the time-dependent response of the data acquisition system to the $B_{1}$-induced signal voltage. Because the frequency is swept linearly through time, the leakage response can also be treated in the frequency domain, which is an intrinsic property of the chirp signal. $h(t)$ can be measured separately in a CW-NMR experiment provided that no MR signal exists in the acquired signal, such as when the sample is removed. The ratio of the frequency sweep range $f_{\mathrm{s}}$ and the total RF duration $t_{\text {acq }}$ is regarded as the frequency sweep rate.

\section{Materials and methods}

\section{Decoupling of RF coils}

All CEA experiments were conducted using a Siemens (Erlangen, Germany) Magnetom 3T clinical MRI system with an eight-channel parallel transmit array unit. The parallel transmit system was used because it allows for an independent control of multiple Tx channels, and in order to facilitate RF supervision of multiple Tx channels the system allows for a concurrent signal reception during RF

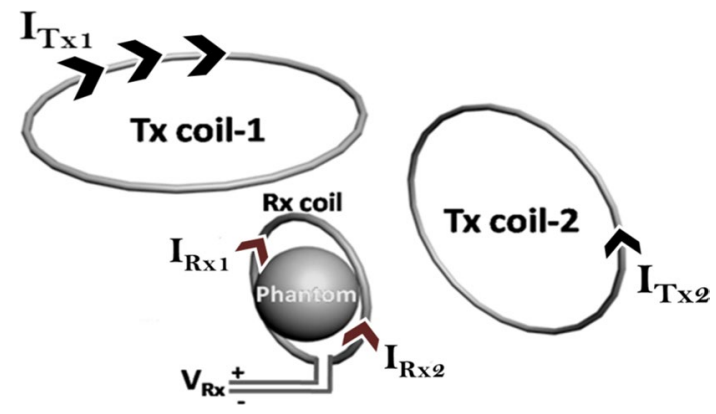

Fig. 1 Visualization of the decoupling process: Tx coil-1 (transmit coil) and the receive coil are placed orthogonally, which reduces the $B_{1}$-induced current on the receive coil. Tx coil- 1 is driven by a current $I_{\mathrm{Tx} 1}$, which induces $I_{\mathrm{Rx} 1}$ on the receive coil. The amplitude and phase of Tx coil-2 (decoupling coil) are adjusted so $I_{\mathrm{Rx} 2}$ cancels $I_{\mathrm{Rx} 1}$ out, which significantly reduces the total $B_{1}$-induced voltage $V_{\mathrm{Rx}}$. Because $I_{\mathrm{Tx} 1}$ is greater than $I_{\mathrm{Tx} 2}$ by the amount of geometrical decoupling, the spins of the sample are excited mostly due to the magnetic field produced by Tx coil-1

excitation, which is not possible with conventional MRI hardware of the same manufacturer.

The coil setup for the CEA experiments consisted of a primary transmit coil (Tx coil-1), a decoupling transmit coil (Tx coil-2), and a receive coil (Rx coil), which is the most simple implementation of the proposed CEA concept (Fig. 1). All RF coils were constructed from adhesive copper tape (3M 1182, 8-mm width) on proton-free glass plates to eliminate the background signals from polymers with very short $\mathrm{T}_{2}$ * values (Fig. 2). The octagon-shaped RF coils have diagonal lengths of 10 and $15 \mathrm{~cm}$ for the Rx coil and the Tx coils, respectively. For 3D MRI experiments, circular RF coils which have diameters of $5 \mathrm{~cm}$ and $10 \mathrm{~cm}$ for the Rx coil and the Tx coils, respectively, are used. All coils are initially tuned to the proton resonance frequency at $3 \mathrm{~T}$ of $123.3 \mathrm{MHz}$. Several measures were taken to maximize decoupling between the Tx coils, and between Tx coil-1 and the Rx coil:

1. Tx coil-1 and -2 are decoupled from each other by capacitive decoupling [25]. Therefore, the coils were connected (see connection between Tx coil-1 and -2 in Fig. 2) and a capacitor was added so that the coupling parameter $S_{12}$ was minimized. With a value $C=88 \mathrm{pF}$, a decoupling of $15 \mathrm{~dB}$ was achieved.

2. Tx coil-1 and the Rx coil were decoupled geometrically by placing the coil planes orthogonally to each other, with the Rx coil at the mid-plane of Tx coil-1 (cf. Fig. 1), which, in the ideal case, nulls the magnetic flux in the Rx coil. In practice, the field polarizations are not ideally linear [26], which limits the achievable amount of decoupling.

3. The remaining $B_{1}$-induced current of Tx coil- 1 in the $\mathrm{Rx}$ coil is cancelled by adjusting the phase and amplitude 
Fig. 2 Schematic of the system setup. The transmit array control unit controls the amplitude and phase as well as the envelopes of the RF waveforms via a modulator. The output of the one of the modulators is amplified by a home-built low noise preamplifier (LNA). An oscilloscope is used to check the voltage levels at the modulator outputs and at the receive coil output in real time. Once the signal voltage level at the receive coil output is reduced to the dynamic range of the receiver circuitry of the scanner, the receive coil is connected to the scanner via a home-built ultra-low noise preamplifier (ULNA)

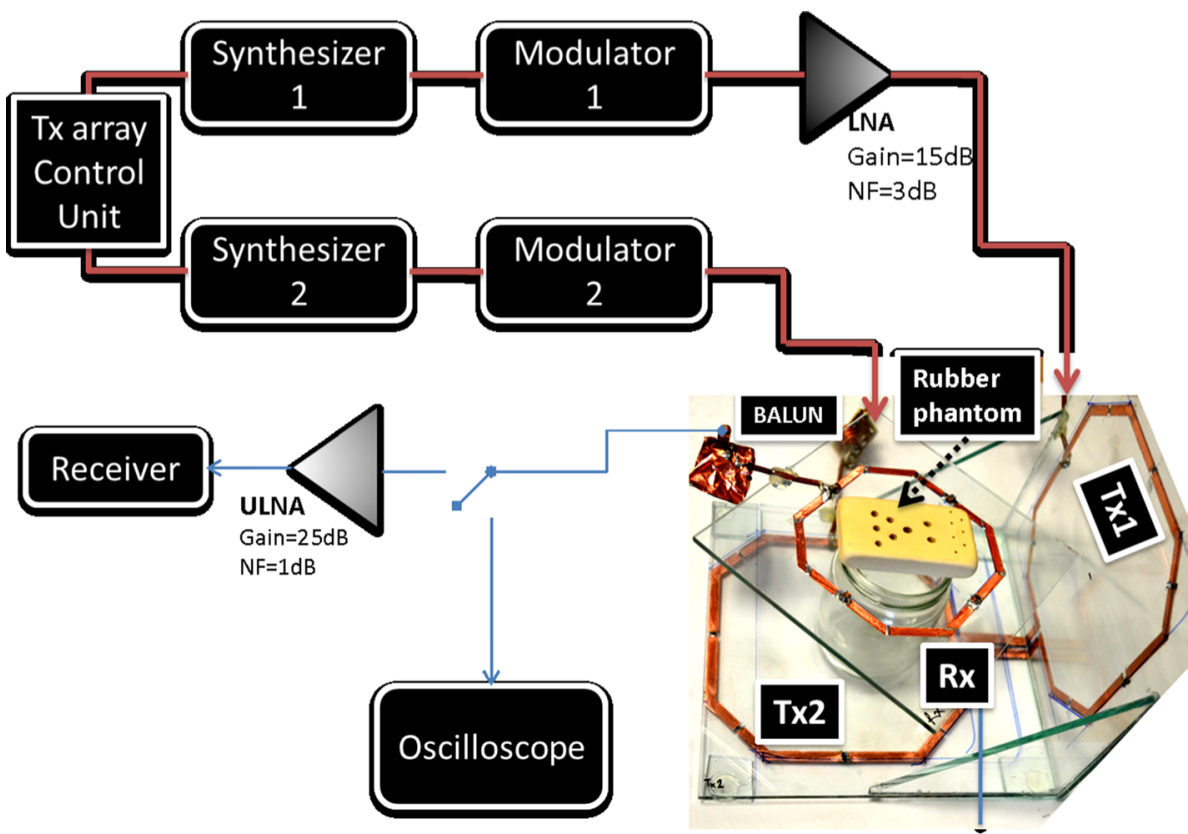

of the transmit field from Tx coil-2 (PA decoupling). Note that the geometrical decoupling between Tx coil-1 and the $\mathrm{Rx}$ coil has already significantly reduced the $B_{1}$-induced transmit signal, and, thus, Tx coil-2 can be driven at an input power level that is much lower than that of Tx coil-1.

4. In addition, the decoupling coil $\mathrm{Tx}$ coil-2 was slightly detuned $\left(\Delta f=2 \mathrm{MHz}, S_{11}(123 \mathrm{MHz})=-3.2 \mathrm{~dB}\right)$ to reduce mutual coupling with the Rx coil. If Tx coil-2 and the Rx coil are weakly coupled, the sensitivity of the $\mathrm{Rx}$ coil is maintained, as no resonance shift occurs.

Due to the low input power applied to Tx coil-2, the flip angle in the sample is mainly determined by Tx coil-1, and the RF transmit field of Tx coil-1 is nearly unchanged. Thus, the magnetization in the sample is still excited by the transmit RF field and an MRI signal can be detected by the Rx coil even during RF excitation. In addition, the orthogonal placement not only reduces the RF signal coupling, but also noise coupling between the RF transmitter and the Rx coil.

Because the commercial RF power amplifier (RFPA) of Tx coil-1 induces high levels of noise up to $400 \mathrm{mV}_{\mathrm{pp}}$, it was replaced by a home-built $100-\mathrm{mW}$ low noise amplifier (LNA) with a gain of $15 \mathrm{~dB}$ and a noise figure of $3 \mathrm{~dB}$. The LNA was designed as a single-stage amplifier using a BFG135 bipolar transistor with input and output matching [27]. Tx coil-2 required much lower RF power levels, and was, thus, directly connected to the modulator output of the second transmit channel (Fig. 2).

The following steps were performed to set up and characterize the coil decoupling:
- To maximize the geometric decoupling, the total unloaded coil setup was placed on the patient table at the iso-center of the magnet. Then the Tx coil-1 was connected to the RF transmitter via the LNA, and the $\mathrm{RX}$ coil was connected to a digital oscilloscope (DSO6104A Agilent). The Rx coil was then slowly rotated, and the amount of decoupling was noted as a function of the relative angle between the coils. The rotation was done first in steps of $5^{\circ}$, which was refined close to the minimum steps of $1^{\circ}$. Then the Rx coil was fixed at the angular position, in which the $B_{1}$-induced voltage was minimal.

- The phantom (see below) was then placed at the imaging volume of the setup for the subsequent PA decoupling calibration to take into account the effect of the conductive sample in the decoupling.

- To adjust the PA decoupling parameters $c$ and $\theta$ from Eq. 2, RF power was individually applied to Tx coil-1, and to Tx coil-2. Then $c$ was calculated from the ratio of the peak voltages, and the phase $\theta$ was first adjusted experimentally via the user interface of the parallel transmit system until the output voltage at the $\mathrm{Rx}$ coil was at the noise level of the oscilloscope output $\left(V_{\mathrm{pp}}=2 \mathrm{mV}\right)$.

- To increase the sensitivity, the Rx coil was then connected to the MR receiver via a home-built ultra-low noise preamplifier (ULNA, gain $25 \mathrm{~dB}$, noise figure $1 \mathrm{~dB}$ ). The decoupling was further improved by fine-adjusting the PA decoupling parameters $c$ (sensitivity $0.001 / 3 \pm 0.5 \mathrm{~dB}$ for a $4-\mathrm{V}_{\text {peak }} \mathrm{RF}$ voltage signal) and $\theta$ (sensitivity $0.01^{\circ} / 3 \pm 0.5 \mathrm{~dB}$ ) iteratively at the Tx array console, until the received signal was close to the noise 
level. The degree of decoupling was plotted as a function of $\theta$ using both the oscilloscope data and the ULNA measurements.

After optimization of the individual decoupling steps, the decoupling schemes were combined to show that CEA is possible in clinical MR systems with minimal modifications.

To provide absolute values for the flip angle, we used Klose's method [28], in which the magnetization was prepared with the chirp pulse, and the remaining longitudinal magnetization was read out as a free induction decay (FID) signal (S1). The experiment was repeated without preparation (S2), and the effective flip angle was calculated from the signal ratio: alpha $=\arccos (\mathrm{S} 1 / \mathrm{S} 2)$.

\section{Transmit noise}

To characterize the noise from the RF transmitter during CEA, RF noise was measured as the standard deviation of the acquired CEA signal from a rectangular RF pulse. Data were acquired for different decoupling conditions: PA decoupling with $20,10 \mathrm{~dB}$ and no geometric decoupling. Signal noise was measured for an RF pulse with a $U=0 \mathrm{~V}$ amplitude and for a coil setup in which the transmit sources are unplugged.

\section{Concurrent excitation and acquisition}

The coil setup was loaded with a cylindrical glass of tap water (glass diameter of $50 \mathrm{~mm}$ ). After the decoupling procedure was carried out as described above, CEA projection data were acquired in the presence of a gradient (Fig. 3). Therefore, a chirp RF pulse of $U_{\text {peak }}=1 \mathrm{~V}$ was applied with a duration of $8.2 \mathrm{~ms}$ and a $8-\mathrm{kHz}$ sweep range, and the gradient amplitude was set to $10 \mathrm{mT} / \mathrm{m}$. Using the $B_{1}$ mapping data, this pulse provides a flip angle of $12^{\circ}$ at the center frequency. The data acquisition was started $100 \mu \mathrm{s}$ after the beginning of the chirp pulse, and 256 data points were sampled over a total period of $8 \mathrm{~ms}$. To be able to subtract remaining background signal from insufficient decoupling, the experiment was repeated without the sample to acquire the system response $h(t)$ (cf. Theory).

During subsequent imaging reconstruction, the reference raw data without a sample were first subtracted from the corresponding data taken with the sample. Then, a deconvolution was performed as outlined in Eq. 3, resulting in radial projection raw data, as would have been acquired with a conventional 2D radial MRI sequence. The deconvolved data were then mapped onto a Cartesian grid using re-gridding, and an inverse Fourier transformation was

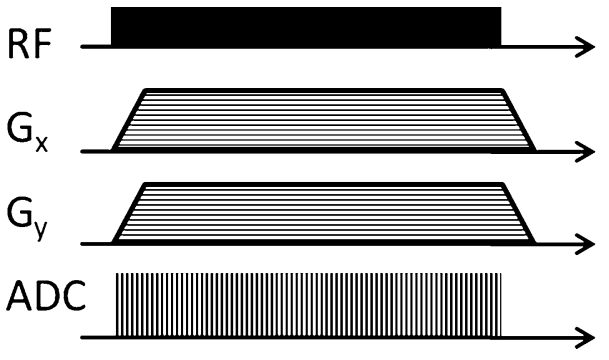

Fig. 3 Pulse sequence diagram for a single TR interval. The gradient strength and the sweep rate determine the field of view (FOV) and resolution

applied [29]. Data processing from raw data to the FID and the projection data is presented for a water sample in Fig. 6.

In a second experiment, the coil setup was loaded with a thin rubber sample $\left(5 \times 3 \times 0.8 \mathrm{~cm}^{3}\right)$, which was chosen because it has a very short $\mathrm{T}_{2} *$ of $500 \mu \mathrm{s}$ (measured independently). Several holes (diameters $0.1-0.4 \mathrm{~cm}$ ) were drilled into the sample to provide an inner structure. The acquisition was repeated by rotating the gradient direction radially over 768 angular values (2D radial sampling), resulting in a total acquisition time of $768 \times 200 \mathrm{~ms}=2: 34 \mathrm{~min}: \mathrm{s}$. An image of the rubber phantom was reconstructed from the CEA data as described above.

CEA pulse sequence was modified to employ a 3D radial k-space trajectory [30]. Ex vivo adipose APOE mouse (65 g body weight) was imaged to demonstrate that the setup can also be used with more realistic objects. The setup was improved with $\mathrm{HD} 24388$ (gain $23 \mathrm{~dB}$, noise figure $0.7 \mathrm{~dB}$ ) and HD29980 (gain $36 \mathrm{~dB}$, noise figure $1.0 \mathrm{~dB}$ ) amplifiers (HD Communications Corp. Ronkonkoma, NY) at both transmit channels. 100,000 radial spokes were acquired during chirp RF excitation spanning $40 \mathrm{kHz}$ over $4 \mathrm{~ms}$. In time interleaved mode, this pulse produced a $9^{\circ}$-flip angle at a homogenous phantom. Maximum gradient strength was set to $24 \mathrm{mT} / \mathrm{m}$, and 512 points per spoke were acquired. Spoiler gradients of $24 \mathrm{mT} / \mathrm{m}$ amplitude and $4 \mathrm{~ms}$ duration were applied following each acquisition. A TR of $20 \mathrm{~ms}$ resulted in total acquisition time of $33 \mathrm{~min}$. The image was reconstructed onto a $512 \times 512 \times 512$ Cartesian grid using a Gaussian gridding kernel. For anatomical reference, a 3D GRE sequence with $\mathrm{FOV}=120 \mathrm{~mm}$, $\mathrm{TR}=7 \mathrm{~ms}, \mathrm{TE}=2.21 \mathrm{~ms}$, flip angle $=30^{\circ}$, a $400-\mathrm{Hz} / \mathrm{Px}$ bandwidth, a $0.4-\mathrm{mm}$ resolution and a $0.5-\mathrm{mm}$ slice thickness was applied.

The effect of the transmit system instability on the acquired signal after decoupling was also demonstrated by a simple CEA experiment. The $B_{1}$-induced leakage signal was acquired at 1-min steps while keeping the entire 
sequence parameters constant using a CEA setup with no sample present.

\section{Results}

\section{Decoupling of RF coils}

Geometric decoupling reduced the induced voltages in the receive coil from $80 \pm 5$ to $8 \pm 1 \mathrm{mV}_{\mathrm{pp}}$, and PA decoupling further decreased the voltage to less than $10 \mu \mathrm{V}_{\mathrm{pp}}$. Thus, with the geometric decoupling alone, an isolation of $18 \pm 3 \mathrm{~dB}$ was achieved, and PA decoupling additionally yielded an isolation of $52 \pm 5 \mathrm{~dB}$. In total, a decoupling of $70 \pm 8 \mathrm{~dB}$ was possible with the proposed setup. For chirp pulses, PA decoupling deviated $10 \mathrm{~dB}$ over a $30-\mathrm{kHz}$ bandwidth. The resulting plot of the geometric decoupling is shown in Fig. 4, and the plot for the PA decoupling using the multichannel transmission system is shown in Fig. 5. Voltage levels and decoupling values are given as an average over 60 repeated measurements.

From the $B_{1}$ mapping experiment, the $B_{1}$ magnitude of the rectangular RF pulse in the CEA experiment was calculated as $0.1 \mu \mathrm{T}$ for a rectangular RF pulse of an 8-ms duration and a $U_{\text {peak }}=1 \mathrm{~V}_{\mathrm{pp}}$. Note that $B_{1}$ was calculated only for Tx coil-1.

\section{Transmit noise}

Without geometric decoupling, the standard deviation of the acquired CEA signal was $14 \mu \mathrm{V}$; with 10 - and $20-\mathrm{dB}$ geometric decoupling, the standard deviation was 11 and $3 \mu \mathrm{V}$, respectively. The acquired signal voltage levels were the same when the transmit system was driven with an RF pulse with a zero amplitude, and when the transmit system was turned off and unplugged. Therefore, it was concluded that the transmit noise-induced voltage does not exceed the receive noise floor which is measured as $5 \mu \mathrm{V}_{\mathrm{pp}}$.

\section{Application to MRI with CEA}

Figure 6 shows the acquired raw data processed through leakage subtraction, a smoothing filter, and deconvolution. Contamination from a $B_{1}$-induced leakage signal can be observed as a frequency-dependent DC offset in the raw data with the sample (Fig. 6a). $B_{1}$-induced leakage $A(\mathrm{t})$ is
Fig. 4 Coupling versus angle between coil planes for a $180^{\circ}$ rotation of the receive coil with respect to the Tx coil. An ideal linearly polarized field is also calculated, and both fields are scaled around $0^{\circ}$
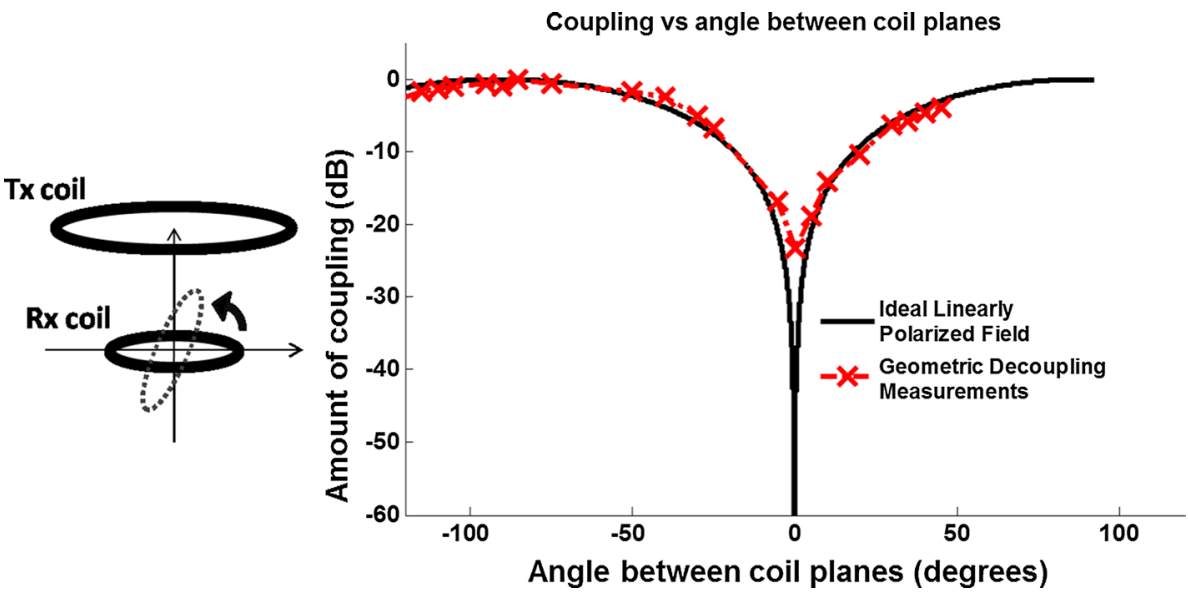

Fig. 5 Coupling versus phase of an RF generator unit. Phase and amplitude (PA) decoupling is represented by changing the phase of one Tx channel step by step over $360^{\circ}$. The data are scaled around $0^{\circ}$
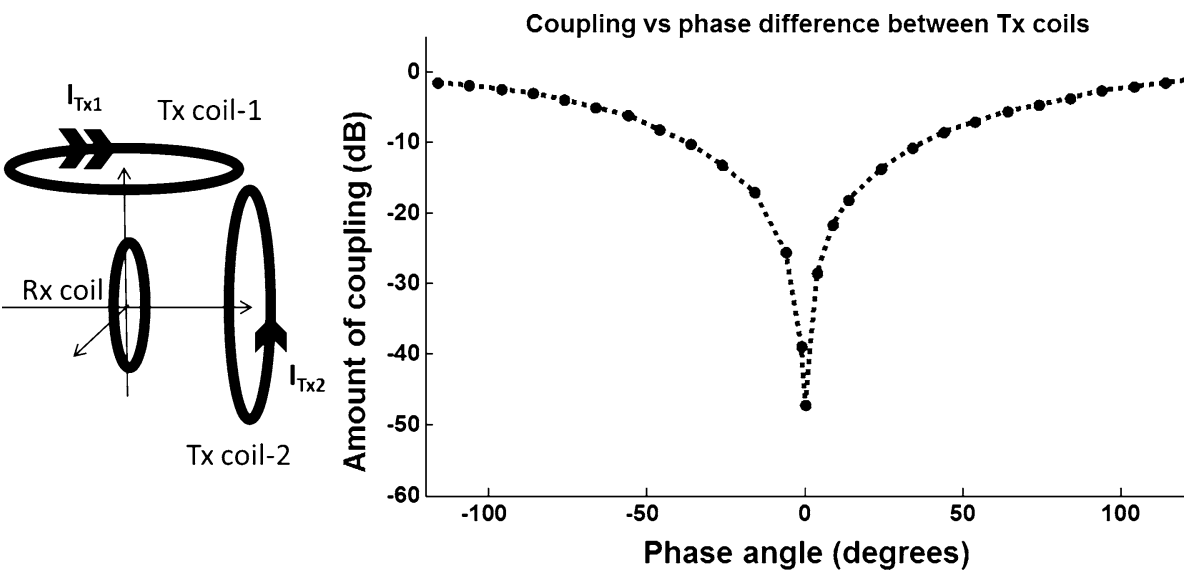

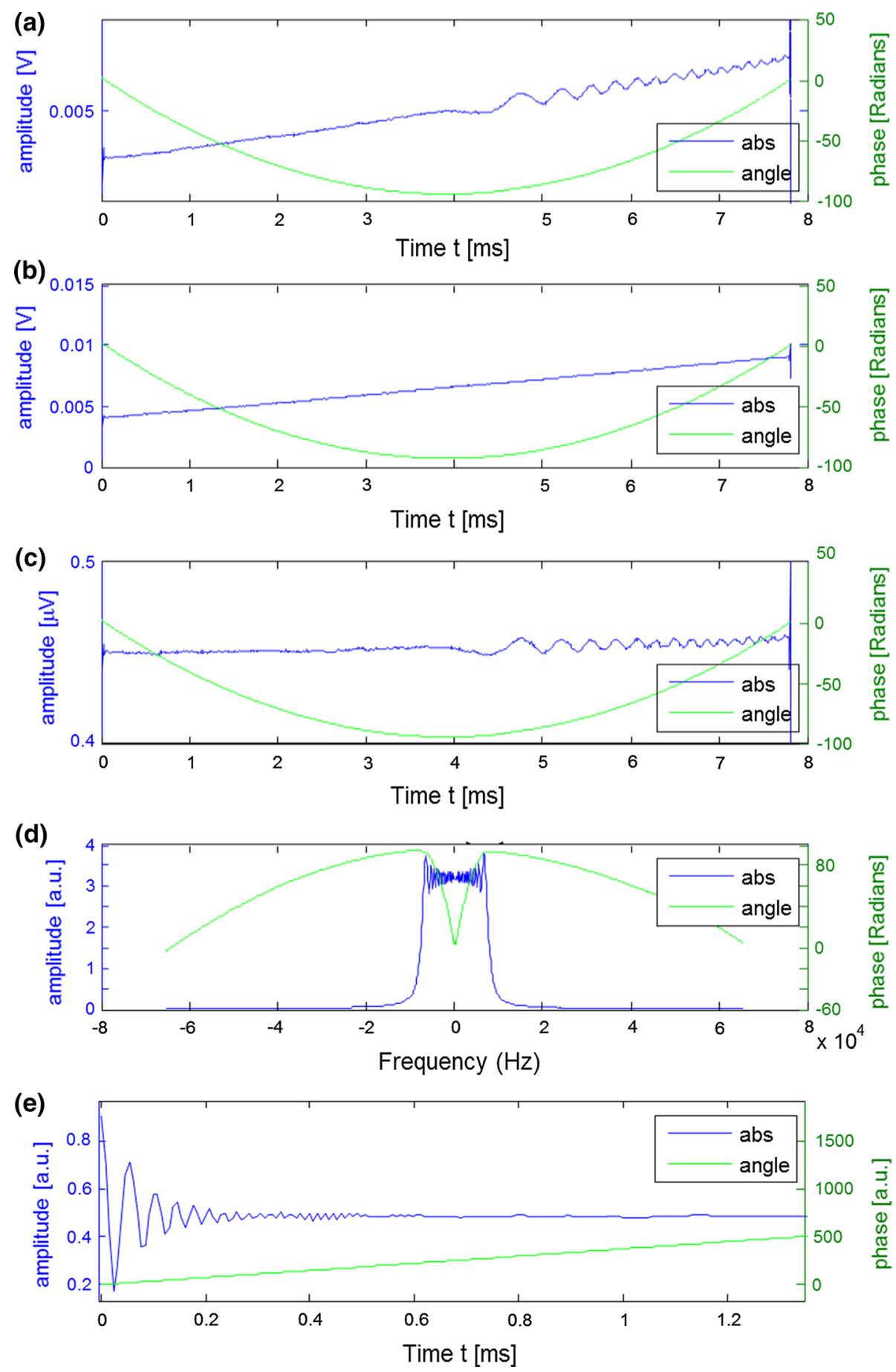

Fig. 6 a Raw data acquired from a water sample in the presence of a $10-\mathrm{mT} / \mathrm{m}$ gradient field along the $x$ direction: $s(t)=F I D \otimes B_{1}(t)+A(t)$. b Measured input $B_{1}$ waveform as a chirp function representing a leakage signal that is convolved by the system's response function: $A(t)=h(t) \otimes B_{1}(t)$. c Acquired

measured by repeating the same acquisition after the sample is removed (Fig. 6b). Subtraction of the waveforms results in the signal waveform without contamination, as is shown in Fig. 6c. The remaining DC offset in the data signal after the leakage subtraction: $s(t)-A(t)=F I D \otimes B_{1}(t)$. d Fourier transform of the estimated FID signal after deconvolution: $\mathcal{F}(\widetilde{F I D})=\mathcal{F}(s(t)-A(t)) / \mathcal{F}\left(B_{1}(t)\right)$. e Single-sided FID signal, which is an approximation of the actual FID: FID

was also subtracted before the next step. Deconvolution was then performed in the Fourier domain using the analytical expression for the chirp pulse $B_{1}(t)$ from Eq. 3 as the deconvolution kernel yielding a radial projection of the data 
Fig. 7 a Rubber phantom with a $\mathrm{T}_{2}{ }^{*}$ value of $500 \mu \mathrm{s}$ with holes, b CEA image with a base resolution of 256 and 768 radial spokes; TR $=200 \mathrm{~ms}$. There is blurring at the edges, but the holes of various dimensions $(0.1-0.4 \mathrm{~mm})$ drilled on the rubber are clearly visible
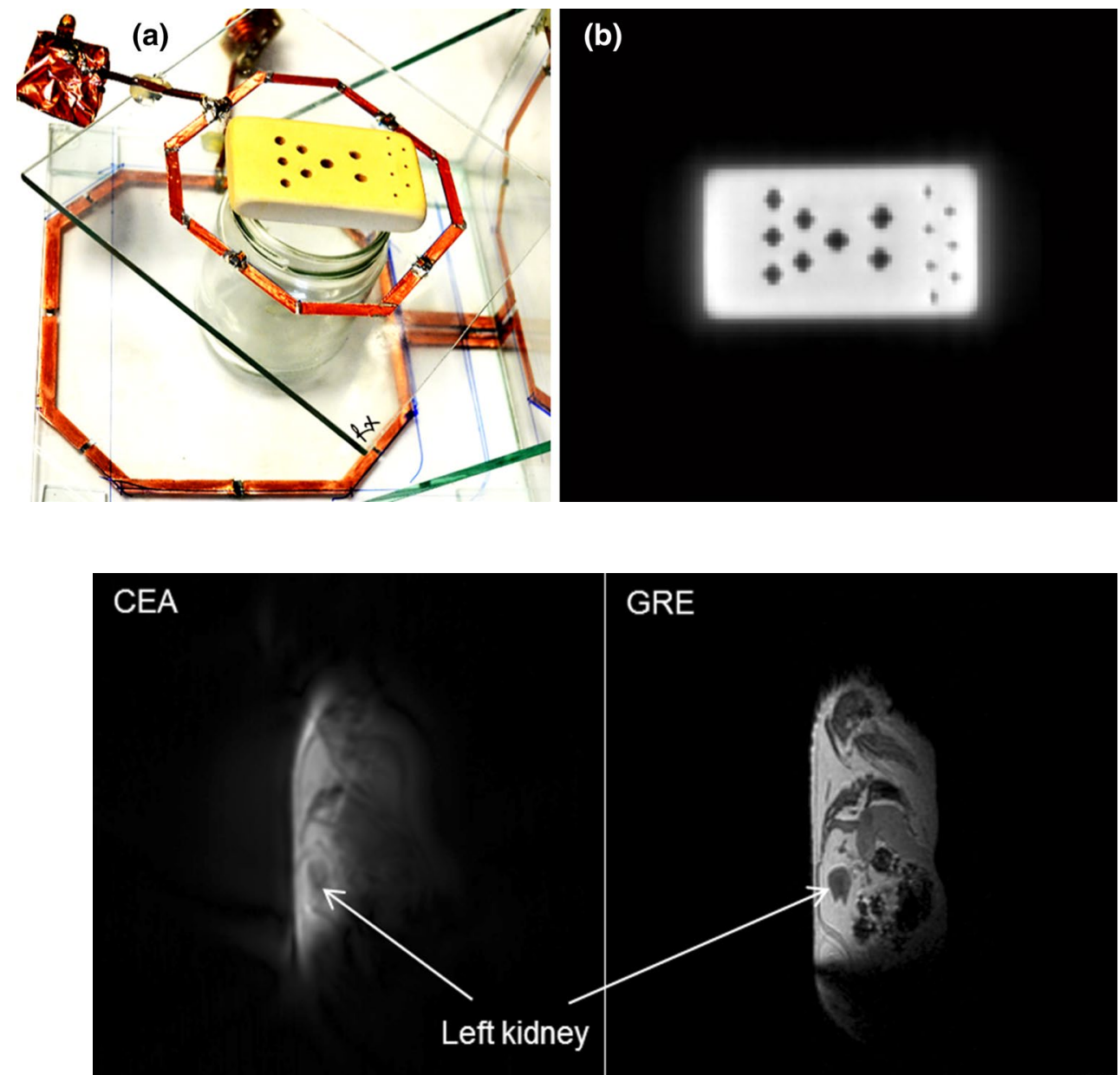

Fig. 8 Sagittal slices from 3D MRI of ex vivo APOE mouse with CEA and GRE. GRE image shows all the anatomical details with a 0.4-mm in-plane resolution. Major structures are visible in the CEA image, such as the left kidney
(Fig. 6d). For gridding reconstruction, an inverse Fourier transform was performed to obtain the frequency-encoded FID signal (Fig. 6e). The reconstructed CEA image after re-gridding is shown in Fig. 7. The rubber phantom with all its inner structures is clearly visible, and some blurring is observed at the edges of the phantom.

Center slices of 3D GRE and CEA data are shown for a sagittal plane in Fig. 8. The anatomical details are available in GRE images. The artifacts and the blurring together result in loss of significant anatomical details, yet the major structures are visible in the first proof-of-principle 3D CEA image slice. The left kidney of the mouse is pointed and visible in both images. The contrast properties of the CEA image require further analysis.

The unstable nature of the RF transmit system is observable in Fig. 9 even with the achieved decoupling during RF excitation. In acquisition of each spoke, there would be minor deviations in the $\mathrm{B}_{1}$-induced leakage signal.

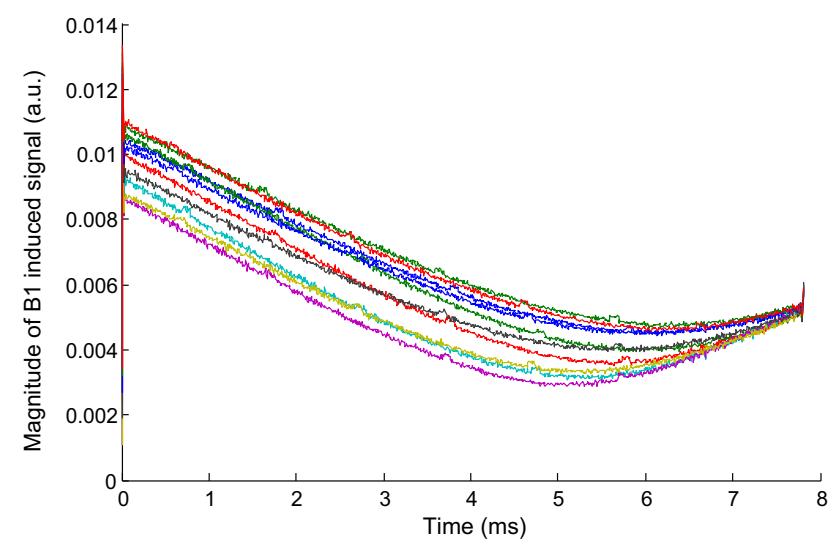

Fig. 9 Variation of $B_{1}$-induced leakage signal over subsequent measurements after decoupling. Due to the deviations in the leakage signal, the resulting images are prone to artifacts since the leakage signal is assumed to be constant during the acquisition of the entire k-space 


\section{Discussion}

In the past, hybrid coupler decoupling was proposed by Idiyatullin and coworkers to reduce $B_{1}$-induced signal leakage in CEA MRI [15]. In their work, they achieved a maximal decoupling of $40 \mathrm{~dB}$, which necessitated the use of additional subtraction techniques to be able to reconstruct meaningful MR images. In this work, higher decoupling was achieved; however, a subtraction was still needed to remove the remaining $B_{1}$-induced leakage signals. Nevertheless, the increased decoupling in this work provides a better use of the dynamic range of the receiver, which will translate directly into an increased image dynamic range. Note that the dynamic range can be traded off against sweep rate in chirped acquisitions, and, thus, the dynamic range per unit time could be used as a figure of merit for CEA acquisitions.

In this work, two subsequent data sets were acquired: One with the regular experimental setup and the other with the sample removed. Measuring the signal with an unloaded coil is a commonly applied technique [9], although the leakage signal has also been described analytically in classical CW-NMR experiments [31]. This analytical approach would be advantageous for clinical applications since the additional measurements without coil loading can be avoided.

Minimization of the transmission noise is an important factor during CEA experiments. To minimize RF transmit noise, the conventional RF power amplifiers with $400 \mathrm{mV}_{\mathrm{pp}}$ noise voltage were replaced with an LNA. The noise voltage of this LNA was less than $2 \mu \mathrm{V}_{\mathrm{pp}}$; thus, for these experiments transmit noise was not the dominant factor in the acquired CEA signal. In general, a CEA system should always use very low-noise transmit systems, which might require replacing some components in the transmit chain of clinical MRI systems. The ultimate aim of providing transmit-receive isolation over $100 \mathrm{~dB}$ requires demanding hardware modifications as well. This includes employing amplifiers with flat gain over the excitation bandwidth, providing constant noise figures of less than $0.5 \mathrm{~dB}$; additionally the phase-locked-loop (PLL) clock multiplier phase noise of the digital-to-analog converter modules, phase noise and jitter of the reference oscillators of the synthesizers should be minimized [3234] using high performance products of RF communication electronics, such as yttrium-iron-garnet oscillators [35] and high electron mobility transistors [36]. In addition, advanced spurious signal control is required so that the overall noise floor of the signal generator unit is kept as low as possible. For experiments in humans, higher transmit powers are required, which would lead to an increase in the noise floor, if these experiment would simply be upscaled. However, using multiple small transmit coils with LNAs, as was done in this experiment, might yield sufficiently low noise floors-this will be investigated in future experiments.

In PA decoupling, different RF transmit systems are used. These RF sources have uncorrelated noise characteristics, and it is, therefore, not possible to decouple them with PA decoupling alone. In this work an orthogonal coil placement was therefore used that provides an additional decoupling mechanism. Another concept would be to reintroduce noise correlation by using a single RF source and later split the RF power to both transmit coils Tx coil-1 and -2 . This would require a very stable RF amplifier, and additional phase and amplitude control units, but it would offer the opportunity to use the PA concept on every conventional MRI system without parallel RF transmitters.

To use the PA decoupling in clinical applications the PA decoupling must be stable over the total acquisition of the images. Decoupling instabilities could originate, for example, from transmit voltage fluctuations, frequency drifts in the modulators, phase noises based on oscillator infidelities, and time-varying cable interferences. These system instabilities can lead to time-varying leakage signals, so that a reacquisition of the subtraction signal $A(t)$ might be required. This could be difficult to achieve in a clinical application, and, thus, it needs to be investigated as to whether the original time course $A(t)$ can be adapted numerically (e.g., by scaling) to small changes in the decoupling. In these pilot experiments, the acquisition time for a CEA data set was more than 2 min due to the relatively long repetition time of $200 \mathrm{~ms}$, which was chosen to maximize the MR signal. In a clinical routine, much shorter TRs are used, so that CEA MRI measurements will become less susceptible to long term fluctuations of the decoupling, which are demonstrated in Fig. 9. If the $B_{l}$-induced leakage deviates at each acquired spoke, the resulting k-space would be contaminated by undefined frequency modulations which might result in artifacts effecting low or high frequency information content. Therefore, a more comprehensive treatment of $B_{1}$-induced leakage subtraction is necessary in advance to further applications of the method. For large bandwidth applications (i.e., in vivo), it is important that the decoupling over the excitation bandwidth is sufficiently high to keep the signal within the dynamic range of the receiver.

During patient motion, PA decoupling may be variable, as was reported for the change of the coil s-parameters and impedance due to breathing motion [37-39]. These motioninduced load changes were on the order of a few percent, which would not exceed the dynamic range of the receiver in our experiment. The difference in isolation between two extreme cases of a fully loaded coil and an unloaded coil was less than $20 \mathrm{~dB}$, and the leakage signal was still within the receiver dynamic range. Nevertheless, a real time monitoring of the loading (as was discussed in [40]) along with 
a feedback system would definitely increase the robustness of the active decoupling system against motion.

In this work, we used custom-designed RF amplifiers. Even though care was taken to operate them in their linear regime, non-linearities cannot be fully excluded. In commercial MR systems, RF non-linearities are compensated by calibration measurements and pre-emphasis, which is also possible with this implementation. Note, however, that small RF transmit non-linearities do not affect the deconvolution (and, thus, the image quality) as they are intrinsically accounted for by the measurement of $B_{1}$. For the PA decoupling, transmit nonlinearities might lead to a less optimal isolation, in particular for RF pulses with a large dynamic range. In this case, decoupling would be dependent on the current RF pulse amplitude and phase, which varies over pulse.

In the reconstructed images, blurring of the edges can be observed. Radial sampling and $\mathrm{T}_{2} *$ decay limits the fullwidth at half-maximum (FWHM) resolution [15]. For the rubber sample with a very short $\mathrm{T}_{2} *$ of $0.5 \mathrm{~ms}, \mathrm{a} \mathrm{T}_{2} *$ decaydependent blurring of eight pixels was expected. Additional blurring would be introduced due to the large effective slice thickness caused by 2D acquisition without slice selection, $\mathrm{B} 0$ at the edges, and the angle between the holes axes and the imaging plane. If we assume a short $\mathrm{T}_{2}{ }^{*}$ of $10 \mathrm{~ms}$ (which is already shorter than typical $\mathrm{T}_{2}{ }^{*} \mathrm{~s}$ in tissue at $3 \mathrm{~T}$ ), the $\mathrm{T}_{2}{ }^{*}$ decay during data acquisition would lead to a widening of the point spread function FWHM by a factor of 0.2 - thus, the $\mathrm{T}_{2}{ }^{*}$ decay and 3D sampling cannot account for the observed blurring of more than two voxels. The blurring could be caused by small errors in the gradient trajectories which lead to misalignments of the k-space data. Another possible source of blurring could be a filtering effect in k-space: depending of the location in the imaged object, the actual time of the excitation within the sweep pulse varies, which leads to a spatially varying repetition time. This effect is small from one radial projection to the next, but it can introduce variable $\mathrm{T}_{1}$ relaxation and, thus, amplitude modulation. To improve image quality, gradient trajectories need to be mapped, and the k-space filtering effect could be removed by advanced pulse sequence strategies. Increased bandwidth is a direct way to decrease off-resonance blurring in radial imaging of fast relaxing spins, where the trend of increasing the maximum gradient strength will help to improve CEA MRI.

In this work the position dependence of $B_{1}$ was not included in the analysis described by Eq. 3. As the rubber sample was smaller than the Tx coil, this $B_{1}$ inhomogeneity is not expected to influence the quality of the result significantly. Note further, that in the CEA experiment, a frequency-swept RF pulse was used, which is similar to an adiabatic pulse for spin excitation [41]. Adiabatic pulses provide nearly homogeneous RF excitation even in the presence of inhomogeneous $B_{1}$, and, thus, the CEA experiment can be further improved by a careful selection of an adiabatic excitation pulse. With adiabatic pulses, the small tip angle approximation is violated, but a reconstruction should still be possible, as the response of the spin system to the excitation pulse can be computed numerically.

The PA concept can be further expanded by the use of more than two transmit coils that would potentially reduce the remaining $B_{1}$-induced voltage after PA decoupling. Unfortunately, these additional coils can couple to the other transmit coils which might reduce the decoupling efficiency. Numerical simulations or approximate analytical solutions are required to analyze this decoupling problem, which is beyond the scope of the present study. Nevertheless, decoupling with two Tx coils is already good enough to provide CEA MR images with minimal $B_{1}$ leakage. Another alternative might be a direct Cartesian feedback of the transmit signal as proposed by Hoult et al. [42]. PA decoupling can potentially be combined with other decoupling methods to achieve extra isolation.

At higher field strengths, PA decoupling can be implemented theoretically using the same approach presented here. However, at higher field strengths, coil and sample dimensions are closer to the RF wavelength, the coupling between $\mathrm{Tx}$ and Rx coil may vary more strongly at small displacements of the sample (e.g., during breathing). Nevertheless, the decoupling is still scalar, and the problem is conceptually similar to $B_{1}$ inhomogeneity correction with parallel transmit systems. Here, the $B_{1}$ map of each transmit coil is measured and the optimum phase/amplitude modulation parameters are calculated to minimize the induced voltage in the receive coil, whereas in $B_{1}$ inhomogeneity correction the $B_{1}$ field in the target area is made homogeneous. Thus, all existing technologies for $B_{1}$ homogenization can be adapted, ranging from simple $B_{1}$ shimming to the individual modulation of the RF pulse shapes for each transmit channel to achieve maximum transmit-receive isolation.

As a further improvement, it is possible to use an optimized RF pulse at Tx coil-2 so that the decoupling is maximized. Such a pulse could take into account additional effects such as system non-linearities, and it would bring more degrees of freedom to the setup.

The same setup and system can also be used for conventional pulsed MR sequences such as UTE. The concept of reducing the $B_{1}$-induced currents in UTE sequences was first presented as a potential solution to the acquisition delay problem in UTE sequences due to coil ring down time [23]. The acquisition delay after RF excitation is caused by T/R switching, ADC filtering, and the coil ring down time. Using the active decoupling method presented in this work, $\mathrm{T} / \mathrm{R}$ switches can be eliminated from the setup because the method provides an isolation of $70 \mathrm{~dB}$ between 
the transmit and receive coils with the initial setup of two transmitting and one receive coils. In addition, this isolation decouples the Tx and Rx coils during the excitation, resulting in a reduction in the energy that is deposited in the receive coil during excitation and a decrease in coil ring down time. Therefore, this decoupling method is a potential solution for the acquisition delay problem in UTE sequences.

Extension of the presented decoupling scheme to a planar surface array instead of a single Rx loop coil is challenging to implement. Initially we can start with a planar surface array and treat signals from each coil element separately. Afterwards, signals from all receive elements could be combined. Geometric decoupling is possible for all the coil elements and a certain amount of decoupling could be achieved for each element, although not optimally. It could also be the case that the system requires at least an equal number of transmitters and would still leave the question unanswered, if the field produced by the transmitter array, while fully and actively decoupling it from the receiver, would still be efficient for spin excitation. Extension to the receive arrays is problematic at this point of the research. Experimental validation is required to take a further step towards CEA MRI with receive arrays.

State-of-the-art gradients of clinical MRI systems offer high maximum gradient strengths (up to $80 \mathrm{mT} / \mathrm{m}$ ) and high slew rates (typically up to $200 \mathrm{~T} / \mathrm{m} / \mathrm{s}$ ). Combined with fast $T / R$ switching circuits designed specifically to reduce acquisition delays [3, 13], high performance gradients can already achieve very broad bandwidths that cover extremely short $\mathrm{T}_{2}{ }^{*}$ components in vivo. However, rapid gradient switching is limited by physiology due to peripheral nerve stimulation [43], and increasing gradient strengths are more demanding in terms of system engineering. CEA, on the other hand, approaches the problem from a different perspective and reduces a few hardware demands such as the need for very fast switching circuits. In addition, CEA can evolve in line with parallel transmit array systems. Further studies are necessary to investigate the benefits of CEA in vivo and provide a sound comparison between state of art techniques for imaging of tissue with ultrashort $\mathrm{T}_{2}$ *

\section{Conclusion}

In this work a new method to decouple transmit and receive systems was described for CEA using a clinical MRI system with a parallel transmit array system. With this method, a decoupling of more than $70 \mathrm{~dB}$ was achieved. In a phantom experiment, CEA MRI could be demonstrated in a rubber phantom with sub-millisecond $\mathrm{T}_{2} *$ relaxation times. 3D adaption of a CEA sequence was also demonstrated on an ex vivo animal as a more realistic sample and as an initial step prior to in vivo applications.

Currently, different MRI techniques are investigated for short $\mathrm{T}_{2} *$ tissues such as solids and tightly bound water in bones. For this, many short $\mathrm{T}_{2} *$ imaging methods have been suggested such as UTE [44], ZTE [3], SWIFT [13], WASPI [45], or SPRITE [6]. CEA is considered an alternative to these methods, but it has not been investigated in wholebody MR systems due to the challenges in implementation, some of which are reviewed in this study. CEA would be advantageous as it requires a much lower peak power, and it truly provides a zero echo time. However, the decoupling method described here is not restricted to CEA applications. Rather, it can be applied in all applications where $B_{1}$ induced currents need to be decreased without changing the sensitivity of the transmit field.

\section{Compliance with ethical standards}

Conflict of interest The authors declare that they have no conflicts of interest.

Human and animal rights This article does not contain any studies with human participants or animals performed by any of the authors.

\section{References}

1. Gatehouse P, Bydder G (2003) Magnetic resonance imaging of short T2 components in tissue. Clin Radiol 58(1):1-19

2. Tyler DJ, Robson MD, Henkelman RM, Young IR, Bydder GM (2007) Magnetic resonance imaging with ultrashort TE (UTE) pulse sequences: technical considerations. J Magn Reson Imaging 25(2):279-289

3. Weiger M, Brunner DO, Dietrich BE, Muller CF, Pruessmann KP (2013) ZTE imaging in humans. Magn Reson Med 70(2):328-332

4. McDonald PJ (1997) Stray field magnetic resonance imaging. Prog Nucl Magn Reson Spectrosc 30:69-99

5. Emid S, Creyghton JHN (1985) High resolution imaging in solids. Phys B 128:81-83

6. Balcom BJ, Macgregor RP, Beyea SD, Green DP, Armstrong RL, Bremner TW (1996) Single-point ramped imaging with T1 enhancement (SPRITE). J Magn Reson A 123(1):131-134

7. Dadok J, Sprecher RF (1974) Correlation NNW spectroscopy. J Magn Reson 13(2):243-248

8. Gupta RK, Ferretti JA, Becker ED (1974) Rapid scan Fourier transform NMR spectroscopy. J Magn Reson 13(3):275-290

9. Lurie DJ, McCallum SJ, Hutchison JMS, Alecci M (1996) Continuous-wave NMR imaging of solids. MAGMA 4:77-81

10. Davies GR, Lurie DJ, Hutchison JMS, McCallum SJ, Nicholson I (2001) Continuous-wave magnetic resonance imaging of short T-2 materials. J Magn Reson 148:289-297

11. Fagan AJ, Davies GR, Hutchison JM, Lurie DJ (2003) Continuous wave MRI of heterogeneous materials. J Magn Reson 163(2):318-324

12. Fagan AJ, Davies GR, Hutchison JM, Glasser FP, Lurie DJ (2005) Development of a 3-D, multi-nuclear continuous wave NMR imaging system. J Magn Reson 176(2):140-150 
13. Idiyatullin D, Corum C, Park JY, Garwood M (2006) Fast and quiet MRI using a swept radiofrequency. J Magn Reson 181(2):342-349

14. Robson MD, Bydder GM (2006) Clinical ultrashort echo time imaging of bone and other connective tissues. NMR Biomed 19(7):765-780

15. Rahmer J, Bornert P, Groen J, Bos C (2006) Three-dimensional radial ultrashort echo-time imaging with $\mathrm{T} 2$ adapted sampling. Magn Reson Med 55(5):1075-1082

16. Weiger M, Pruessmann KP, Hennel F (2011) MRI with zero echo time: hard versus sweep pulse excitation. Magn Reson Med 66(2):379-389

17. Garwood M (2013) MRI of fast-relaxing spins. J Magn Reson 229:49-54

18. Bloch F, Hansen WW, Packard M (1946) The nuclear induction experiment. Phys Rev 70(7-8):474-485

19. Brunner DO, Dietrich BE, Pavan M, Pruessmann K (2012) MRI with sideband excitation: Application to continuous SWIFT. In: Proceedings of the 20th scientific meeting, International Society for Magnetic Resonance in Medicine, Melbourne, Australia, $p$ 150

20. Idiyatullin D, Suddarth S, Corum C, Adriany G, Garwood M (2011) Continuous SWIFT. In: Proceedings of the 19th scientific meeting, International Society for Magnetic Resonance in Medicine, Montreal, Quebec, Canada. p 382

21. Idiyatullin D, Suddarth S, Corum CA, Adriany G, Garwood M (2012) Continuous SWIFT. J Magn Reson 220:26-31

22. Ozen AC, Ertan NK, Atalar E (2012) Detection of MR signal during RF excitation using a transmit array system. In: Proceedings of the 20th scientific meeting, International Society for Magnetic Resonance in Medicine, Melbourne, Australia, p 2295

23. Ozen AC, Atalar E (2013) Decoupling of transmit and receive coils using a transmit array system: Application to UTE and CEA. In: Proceedings of the 21 st scientific meeting, International Society for Magnetic Resonance in Medicine, Salt Lake City, Utah, USA, p 767

24. Eryaman Y, Akin B, Atalar E (2011) Reduction of implant RF heating through modification of transmit coil electric field. Magn Reson Med 65(5):1305-1313

25. Lee RF, Giaquinto RO, Hardy CJ (2002) Coupling and decoupling theory and its application to the MRI phased array. Magn Reson Med 48(1):203-213

26. Glover G, Hayes C, Pelc N, Edelstein W, Mueller O, Hart H, Hardy C, O'Donnell M, Barber W (1985) Comparison of linear and circular polarization for magnetic resonance imaging. $\mathrm{J}$ Magn Reson 64(2):255-270

27. Ludwig R (2000) RF circuit design: Theory and applications, 2nd edn. Pearson Education, India

28. Klose U (1992) Mapping of the radio frequency magnetic field with a MR snapshot FLASH technique. Med Phys 19(4):1099-1104

29. Jackson JI, Meyer CH, Nishimura DG, Macovski A (1991) Selection of a convolution function for Fourier inversion using gridding [computerised tomography application]. IEEE Trans Med Imaging 10(3):473-478

30. Saff EB, Kuijlaars ABJ (1997) Distributing many points on a sphere. Math Intell 19(1):5-11

31. De Graaf RA (2008) In vivo NMR spectroscopy: principles and techniques. Wiley-Interscience, New York

32. Bahl I, Bhartia P (2003) Microwave solid state circuit design. Wiley-Interscience, New Jersey

33. Hajimiri A, Lee TH (1988) A general theory of phase noise in electrical oscillators. IEEE J Solid-State Circuits 33(2):179-194

34. Lee TH, Hajimiri A (2000) Oscillator Phase Noise: a Tutorial. IEEE J Solid-State Circuits 35(3):326-336

35. Kaa B (2004) A simple approach to YIG oscillators. VHF Commun 4:217-224

36. Mimura T (2002) The early history of the high electron mobility transistor (HEMT). IEEE Trans Microwave Theory Tech 50(3):780-782

37. Graesslin I, Glaesel D, Börnert P, Stahl H, Koken P, Nehrke K, Dingemans H, Mens G, Götze J, and Harvey P (2008) An alternative concept of non-sequence-interfering patient respiration monitoring. In: Proceedings of the 16th scientific meeting, International Society for Magnetic Resonance in Medicine, Toronto, Ontario, Canada p 202

38. Graesslin I, Stahl H, Nehrke K, Harvey P, Smink J, Mens G, Senn A and Börnert P (2009) An alternative concept for nonsequence interfering, contact-free respiration monitoring. In: Proceedings of the 17th scientific meeting, International Society for Magnetic Resonance in Medicine, Toronto, Ontario, Canada. p 753

39. Stang PP, Conolly SM, Santos JM, Pauly JM, Scott GC (2012) Medusa: a scalable MR console using USB. IEEE Trans Med Imaging 31(2):370-379

40. Graesslin I, Krueger S, Vernickel P, Achtzehn J, Nehrke K, Weiss S (2013) Detection of RF unsafe devices using a parallel transmission MR system. Magn Reson Med 70(5):1440-1449

41. Tannus A, Garwood M (1997) Adiabatic pulses. NMR Biomed 10(8):423-434

42. Hoult DI, Kolansky G, Kripiakevich D, King SB (2004) The NMR multi-transmit phased array: a Cartesian feedback approach. J Magn Reson 171(1):64-70

43. Ham CL, Engels JM, van de Wiel GT, Machielsen A (1997) Peripheral nerve stimulation during MRI: effects of high gradient amplitudes and switching rates. J Magn Reson Imaging 7(5):933-937

44. Bergin CJ, Pauly JM, Macovski A (1991) Lung parenchyma: projection reconstruction imaging. Radiology 179:777-781

45. Wu Y, Ackerman JL, Chesler DA, Graham L, Wang Y, Glimcher MJ (2003) Density of organic matrix of native mineralized bone measured by water- and fat-suppressed proton projection MRI. Magn Reson Med 50(1):59-68 